\title{
THE SIMPLY-TYPED PURE PATTERN TYPE SYSTEM ENSURES STRONG NORMALIZATION
}

\author{
Benjamin Wack \\ LORIA \& Université Henri Poincaré, Nancy, France \\ Benjamin.Wack@loria.fr
}

\begin{abstract}
Pure Pattern Type Systems $\left(P^{2} T S\right)$ combine in a unified setting the capabilities of rewriting and $\boldsymbol{\lambda}$-calculus. Their type systems, adapted from Barendregt's $\lambda$-cube, are especially interesting from a logical point of view. Strong normalization, an essential property for logical soundness, had only been conjectured so far: in this paper, we give a positive answer for the simply-typed system.

The proof is based on a translation of terms and types from $P^{2} T S$ into the $\lambda$-calculus. First, we deal with untyped terms, ensuring that reductions are faithfully mimicked in the $\lambda$-calculus. For this, we rely on an original encoding of the pattern matching capability of $P^{2} T S$ into the $\lambda$-calculus.

Then we show how to translate types: the expressive power of System $\mathbf{F} \boldsymbol{\omega}$ is needed in order to fully reproduce the original typing judgments of $\boldsymbol{P}^{2} T S$. We prove that the encoding is correct with respect to reductions and typing, and we conclude with the strong normalization of simply-typed $P^{2} T S$ terms.
\end{abstract}

\section{Introduction}

The $\lambda$-calculus and term rewriting provide two fundamental computational paradigms that had a deep influence on the development of programming and specification languages, and on proof environments. The idea that having computational power at hand makes deduction significantly easier and safer is widely acknowledged (Dowek et al., 2003; Werner, 1994). Many frameworks have been designed with a view to integrate these two formalisms: either by enriching first-order rewriting with higherorder capabilities (Klop et al., 1993) or by adding algebraic features to the $\lambda$-calculus (case expressions with dependent types (Coquand, 1992), a typed pattern calculus (Kesner et al., 1996) and calculi of algebraic constructions (Blanqui, 2001)).

The rewriting calculus, or $\rho$-calculus, by unifying the $\lambda$-calculus and the rewriting, makes all the basic ingredients of rewriting explicit objects, in particular the notions of rule application and result. A rewrite rule becomes a first-class object which can be created and manipulated in the calculus, whereas in works like (Blanqui, 2001), the rewriting remains a bit external to the calculus.

In (Cirstea et al., 2001), a collection of type systems for the $\rho$-calculus was presented, extending Barendregt's $\lambda$-cube to a $\rho$-cube. Later, these type systems have 
been studied deeper for the similar formalism of $P^{2} T S$ (Barthe et al., 2003). Yet, the rewriting calculus has also been assigned some type systems that do not prevent infinite reductions (Cirstea et al., 2004). Thus, strong normalization did remain an open problem for $\boldsymbol{P}^{2} T S$. In this paper, we give a first positive answer to this problem. Since consistency is related to termination, this result makes $P^{2} T S$ a good candidate for a proof-term language integrating deduction and computation at the same level.

The main contributions of this paper are:

- a more recent version of $P^{2} T S$, enhanced with a signature for the types of constants and some corrections on the product rules;

- a concise encoding of pattern matching in the $\lambda$-calculus, which has other potential applications for the encoding of term rewriting systems;

- a translation of the simply-typed system of $P^{2} T S$ into System $F \omega$ emphasizing some particular typing mechanisms of $P^{2} T S$;

- a proof of strong normalization for simply-typed $P^{2} T S$ terms.

This paper is organized as follows. In Section 2, we recall the syntax and the smallstep semantics of $\boldsymbol{P}^{2} T S$. In Section 3, we give an untyped version of the translation, showing how pattern matching is encoded. In Sections 4 and 5, we present the type systems of $\boldsymbol{P}^{2} T S$ and System F $\boldsymbol{\omega}$. In Sections 6 and 7, we give the fully typed translation and we outline a proof of correctness for three important elements of the typed translation: variables, constants and delayed matching constraints. In Section 8, we state the key lemmas used in the full strong normalization proof.

We assume the reader is reasonably familiar with the notations and results of typed $\lambda$-calculi (Barendregt, 1992), of the $\rho$-calculus (Cirstea et al., 2004) and of $P^{2} T S$ (Barthe et al., 2003).

Conventions and notations Generally, the reader can assume that every capital letter denotes an object belonging to $P^{2} T S$, and every small letter denotes an object belonging to the $\lambda$-calculus (except for constants and their arity). For instance, in $P^{2} T S$ : $X, Y, Z$ are variables; $A, B, C$ are terms; $P, Q$ are patterns; $a, f, g$ are constants; $\boldsymbol{\Phi}, \boldsymbol{\Psi}$ are types; $\boldsymbol{\Xi}$ is an atomic type. In System $\boldsymbol{F} \boldsymbol{\omega}: \boldsymbol{w}, \boldsymbol{x}, \boldsymbol{y}, \boldsymbol{z}$ are variables; $t, u$ are terms; $\beta, \gamma$ are type variables; $\sigma, \tau$ are types; $k$ is a kind. Moreover, we will use the notations: $\alpha, \alpha_{i}$ for an arity; $\theta$ for a substitution; $\Gamma, \Delta$ for contexts (mainly in $\left.P^{2} T S\right) ; \Sigma$ for a signature.

Syntactic equivalence of terms will be denoted by $\equiv$. If a substitution $\theta$ has domain $X_{1} \ldots X_{n}$ and $\forall i, X_{i} \theta \equiv A_{i}$, we will also write it $\left[X_{1}:=A_{1} \ldots X_{n}:=A_{n}\right]$. We assume that the signature $\boldsymbol{\Sigma}$ of constants that can be used in $P^{2} T S$ is finite, which is legitimate since a given (finite) term only uses a finite number of constants. Therefore, we will number the constants $f_{1}, \ldots, f_{S}$, where $S$ is the cardinal of $\boldsymbol{\Sigma}$. To denote a tuple of terms $B_{k} \ldots B_{n}$, we will use the vector notation $\vec{B}_{(k . . n)}$, or simply $\vec{B}$ when $\boldsymbol{k}$ and $n$ are obvious from the context. This notation will be used in combination with operators according to their default associativity: for instance, in System $F \omega$, $A \vec{B} \triangleq A B_{1} \ldots B_{n}$ and $\lambda \vec{x} . A \triangleq \lambda x_{1} \ldots \lambda x_{n} . A$. To avoid confusion between symbols, we will use bold $\boldsymbol{\lambda}$ and $\boldsymbol{\Pi}$ for $P^{2} T S$ and roman $\lambda$ and $\Pi$ for System $F \boldsymbol{\omega}$. 


\section{$2 P^{2} T S:$ dynamic semantics}

In this section, we recall the syntax of $P^{2} T S$ and their evaluation rules. The syntax of $P^{2} T S$ extends that of the typed $\lambda$-calculus with structures and patterns (Barthe et al., 2003). Several choices can be made for the set of patterns $P$ : in this paper, we only consider algebraic patterns, whose shape is defined below. The main reason for this restriction is that patterns containing symbols such as $\boldsymbol{\lambda}$ require higher-order matching, which seems difficult to encode in a typed $\lambda$-calculus.

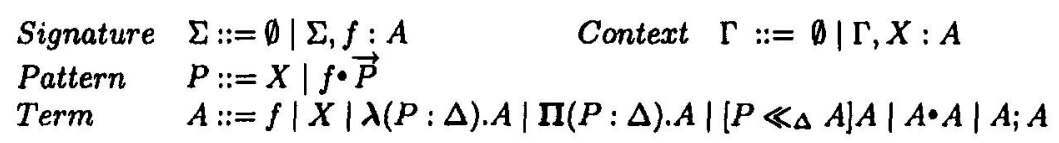

A term with shape $\boldsymbol{\lambda}(P: \Delta) . A$ is an abstraction with pattern $P$, body $A$ and context $\Delta$. The term $[P \ll \Delta B] A$ is a delayed matching constraint with pattern $P$, body $A$, argument $B$ and context $\Delta$. A term $\Pi(P: \Delta) \cdot A$ is a dependent prod$u c t$, and will be used as a type; finally, $(A ; B)$ is a structure and $\boldsymbol{A} \cdot \boldsymbol{B}$ is an application. The application of a constant symbol, say $f$, to a term $A$ will be denoted by $f \cdot A$ too; it follows that the usual algebraic notation of a term is currified, e.g. $f\left(A_{1}, \ldots, A_{n}\right) \triangleq f \cdot A_{1} \cdot \cdots \cdot A_{n} \triangleq f \cdot \vec{A}$.

\section{DEFINITION 1 (FREE VARIABLES $\mathcal{F V}$ OF A TERM)}

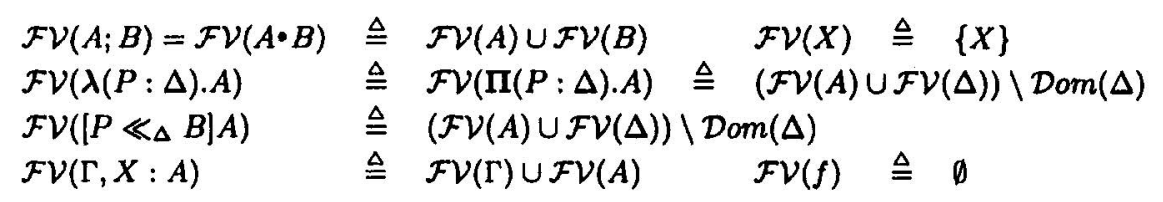

In this paper, extending Church's notation, the context $\Delta$ in $\lambda(P: \Delta) \cdot B$ (resp. $[P \ll \Delta B] A$ or $\Pi(P: \Delta) . B)$ contains the type declarations of the free variables appearing in the pattern $P$, i.e. $\operatorname{Dom}(\Delta)=\mathcal{F} \mathcal{V}(P)$. These variables are bound in the abstraction. The context $\Delta$ will be omitted when we consider untyped terms. As usual, we work modulo $\alpha$-conversion and we use Barendregt's "hygiene-convention" (Barendregt, 1992), i.e. free and bound variables have different names.

For the purpose of this paper, we consider only syntactic pattern matching; a syntactic matching equation $P \ll A$ has either no solution or a unique solution noted $\theta_{(P \ll A)}$. In fact, it seems difficult to encode more elaborated matching theories: for instance, associative matching can generate an arbitrary high number of distinct solutions. Thus, to give a faithful account of all matching solutions in the $\lambda$-calculus, one would probably need a fixed point.
( $\rho)(\lambda(P: \Delta) \cdot A) \cdot B \quad \rightarrow \rho \quad[P \ll \Delta B] A$
( $\sigma) \quad[P \ll \Delta B] A \quad \rightarrow_{\sigma} \quad A \theta_{(P \ll B)}$
$(\delta) \quad(A ; B) \cdot C \quad \rightarrow \delta \quad A \cdot C ; B \cdot C$

Figure 1. Top-level rules of $P^{2} T S$

The top-level rules are presented in Fig. 1. By the $(\rho)$ rule, the application of a term $\lambda(P: \Delta) . A$ to a term $B$ reduces to the delayed matching constraint $[P \ll \Delta B] A$; the 
application of the $(\sigma)$ rule consists in solving the matching equation $P \ll B$ and applying the obtained substitution (if it exists) to the the term $A$. If no solution exists, the $(\sigma)$ rule is not fired and the term $\left[P \ll_{\Delta} B\right] A$ is not reduced. As usual, $\mapsto_{\infty} \delta$ denotes the congruent closure of $\rightarrow_{\rho} \cup \rightarrow_{\sigma} \cup \rightarrow_{\delta}$, and $\mapsto_{a \delta}$ (resp. $==_{\alpha \delta}$ ) is defined as the reflexive and transitive (resp. reflexive, symmetric and transitive) closure of $\mapsto_{\infty} \delta$.

\section{Untyped encoding}

In this section we translate the untyped $P^{2} T S$ with algebraic patterns. The process of syntactic pattern matching consists in discriminating whether the argument begins with the expected constant, and recursively use pattern matching on subterms. It is this (quite simple) algorithm that we encode in the $\lambda$-calculus. We use the following notations: $S$ is the number of symbols appearing in the signature. The $i$ th symbol of $\boldsymbol{\Sigma}$ is denoted by $\boldsymbol{f}_{\boldsymbol{i}}$.

To build the encoding of pattern matching, we need three conditions:

1 each constant $\boldsymbol{f}_{\boldsymbol{i}}$ has a "maximal" arity $\boldsymbol{\alpha}_{\boldsymbol{i}}$, in the sense that $\boldsymbol{f}_{\boldsymbol{i}}$ is never applied to more than $\alpha_{i}$ arguments;

2 in every matching equation $f_{i} \cdot \vec{P}_{(1 . . p)} \ll f_{j} \cdot \vec{B}_{(1 . . q)}$, we have $\alpha_{i}-p=\alpha_{j}-q$;

3 each term $(A ; B)$ has a maximal arity $\boldsymbol{\alpha}$.

In particular, when $i=j$, the second condition reduces to $\boldsymbol{p}=\boldsymbol{q}$, which is an essential condition for resolving this matching equation.

In this section, we assume these properties. In Section 4, we will see that typing enforces the three conditions. They remain true in some untyped situations too: for instance, if we were to encode a Term Rewriting System, the arity of the constants would be given, and partial application of a constant would be forbidden, ensuring that in every matching equation $\alpha_{i}-p=\alpha_{j}-q=0$.

The translation is given in Fig. 2, by a recursive function $\llbracket \cdot \rrbracket$ mapping $P^{2} T S$ terms to $\lambda$-terms. We use a fresh variable $\boldsymbol{x}_{\perp}$; if a closed term is needed, we add an abstraction " $\lambda x_{\perp}$ " once the whole $P^{2} T S$ term is translated.

$$
\begin{aligned}
& \llbracket X \rrbracket \triangleq X \\
& \llbracket f_{i} \rrbracket \triangleq \lambda \vec{x}_{\left(1 . . \alpha_{i}\right)} \cdot\left(\lambda \vec{z}_{(1 . . S)} \cdot\left(z_{i} \vec{x}_{\left(1 \ldots \alpha_{i}\right)}\right)\right) \\
& \llbracket A ; B \rrbracket \triangleq \lambda \vec{x}_{(1 . . \alpha)} \cdot\left(\lambda z \cdot\left(z\left(\llbracket A \rrbracket \vec{x}_{(1 . . \alpha)}\right)\left(\llbracket B \rrbracket \vec{x}_{(1 . . \alpha)}\right)\right)\right) \\
& \llbracket \lambda X . A \rrbracket \triangleq \lambda X . \llbracket A \rrbracket \\
& \llbracket \lambda\left(f_{i} \cdot \vec{P}_{(1 \ldots p)}\right) \cdot A \rrbracket \triangleq \lambda y \cdot\left(y \vec{x}_{\left(p+1 \ldots \alpha_{i}\right)} \overrightarrow{x \perp}_{(1 . . i-1)} \llbracket \lambda \vec{P}_{(1 \ldots p)} \cdot \lambda \vec{x}_{\left(p+1 \ldots \alpha_{i}\right)} \cdot A \rrbracket \vec{x}_{(i+1 \ldots S)}\right) \\
& \llbracket A \cdot B \rrbracket \triangleq \llbracket A \rrbracket \llbracket B \rrbracket \\
& \llbracket[P \ll B] A \rrbracket \triangleq \text { the term obtained by head- } \beta \text {-reducing } \llbracket(\lambda P . A) \cdot B \rrbracket
\end{aligned}
$$

\section{Figure 2. Untyped term translation}

Let us briefly explain this translation:

- In $\llbracket f_{i} \rrbracket$, the variables $x_{1} \ldots x_{\alpha_{\boldsymbol{v}}}$ will be instantiated by the arguments $\vec{B}$ of $f_{i}$ (which explains why we had to bound the arity of $f_{i}$ ). Then, among the variables $z_{1} \ldots z_{S}$, the one corresponding to the head constant of $P$ is selected.

- $\llbracket A ; B \rrbracket$ is translated into the usual pair encoding of the $\lambda$-calculus, and the abstractions $\lambda \vec{x}$ distribute the arguments to both elements of the pair. 
- In $\llbracket \lambda X . A \rrbracket$, the abstraction over a single variable is straightforwardly translated into a $\lambda$-abstraction.

- In $\llbracket \lambda\left(f_{i} \cdot \vec{P}_{(1 . . p)}\right) \cdot A \rrbracket$, the variable $y$ will be instantiated by the argument of this function (for instance $\llbracket f_{j} \cdot \vec{B} \rrbracket$ ). If necessary, the $\boldsymbol{\alpha}_{\boldsymbol{i}}-\boldsymbol{p}$ first occurrences of the variable $x_{\perp}$ instantiate the remaining variables $x_{q+1} \ldots x_{\alpha_{j}}$ which can appear in $\llbracket f_{j} \rrbracket$ : this is where we use the condition $\alpha_{i}-p=\alpha_{j}-q$. Then, if $f_{i}=f_{j}$, the $z_{1} \ldots z_{S}$ select $\llbracket \lambda \vec{P}_{(1 . . p)} \cdot \lambda \vec{x}_{\left(p+1 \ldots \alpha_{i}\right)} \cdot A \rrbracket$ and the encoding of pattern matching can then go on (pointwise) with the sub-patterns $P_{1} \ldots P_{p}$ and the subterms $\boldsymbol{B}_{1} \ldots \boldsymbol{B}_{p}$; if matching fails, $\boldsymbol{x}_{\perp}$ is selected, witnessing the failure. The fresh variables $\boldsymbol{x}_{\boldsymbol{p}+\mathbf{1}}^{\prime} \ldots \boldsymbol{x}_{\boldsymbol{\alpha}_{\boldsymbol{i}}}^{\prime}$ will be instantiated by $\boldsymbol{x}_{\perp}$ 's, but they do not appear in $\llbracket A \rrbracket$. If a variable $X$ has multiple occurrences in the pattern, by $\alpha$ conversion, only one of the subpatterns $\boldsymbol{P}_{\boldsymbol{i}}$ will get the "original" variable, and the other $X$ 's are renamed to fresh variables not occurring in $\llbracket A \rrbracket$ (so matching failures due to non-linearity are not detected by the encoding).

- $\llbracket A \cdot B \rrbracket$ is translated into standard $\lambda$-calculus application.

- $\mathbb{[}[P \ll B] A \rrbracket$ is $\llbracket(\lambda P, A) \cdot B \rrbracket$ where $y$ has been instantiated by $\llbracket B \rrbracket$.

\section{LEMMA 1 (CLOSURE BY SUBSTITUTION)}

For any $\boldsymbol{P}^{\mathbf{2}} \boldsymbol{T} \boldsymbol{S}$ terms $A$ and $\boldsymbol{B}_{\mathbf{1}}, \ldots, \boldsymbol{B}_{n}$, for any variables $\boldsymbol{X}_{\mathbf{1}}, \ldots \boldsymbol{X}_{n}$,

$$
\llbracket A\left[X_{1}:=B_{1} \ldots X_{n}:=B_{n} \rrbracket \rrbracket=\llbracket A \rrbracket\left[X_{1}:=\llbracket B_{1} \rrbracket \ldots X_{n}:=\llbracket B_{n} \rrbracket\right]\right.
$$

\section{THEOREM 1 (F AITHFUL REDUCTIONS)}

For any terms $A$ and $B$, if $A \mapsto_{\infty} B$, then $\llbracket A \rrbracket \mapsto_{\beta} \llbracket B \rrbracket$ in at least one step.

\section{EXAMPLE 1 (TRANSLATION OF A SUCCESSFUL DELAYED MATCHING)}

$$
\begin{aligned}
& \left(\lambda Y . \underline{(\lambda(f \bullet X) \cdot X) \cdot Y)} \cdot(f \bullet a) \mapsto \quad \frac{(\lambda Y .[f \bullet X \ll Y] X) \bullet(f \bullet a)}{(Y \ll f \bullet a)[f \cdot X \ll Y] X}\right. \\
& \mapsto \quad \overline{Y Y \ll f \bullet a][f \cdot X \ll Y] X} \\
& \mapsto \quad\left[f^{\circ} \overline{X \ll f} \cdot a\right] X \\
& \mapsto a
\end{aligned}
$$

The inner delayed matching constraint is essential here because it has to "wait"for the instantiation of $Y$ before performing matching. For the translation, we consider $\Sigma=\left\{a_{1}, f_{2}\right\}$ with $\alpha_{1}=0$ and $\alpha_{2}=1$. The reductions are shown on Fig. 3. The selected $\lambda$-abstraction and its argument are underlined.

\section{The typed $P^{2} T S$ : static semantics}

This section presents a version of the type systems of $\boldsymbol{P}^{2} \boldsymbol{T S}$ with some minor adaptations. The inference rules are given in Fig. 4. For a detailed explanation of these rules, the reader can refer to (Barthe et al., 2003); here, we will only discuss some differences with regard to previous type systems for the $\rho$-calculus and $P^{2} T S$ :

- In (Cirstea and Kirchner, 2000), a first strongly normalizing type system for the $\rho$-calculus was introduced; however, the proof of normalization is mainly based on a heavy restriction over the types of constants. 


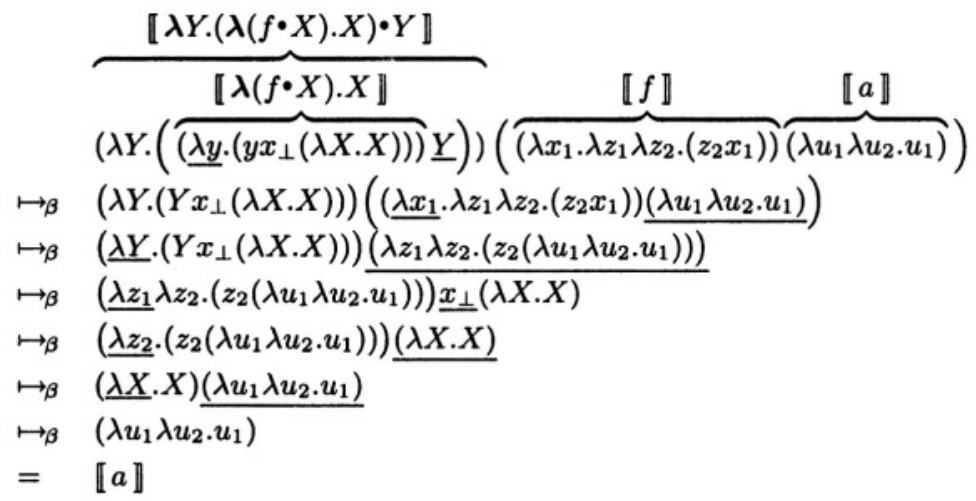

Figure 3. Translation of a successful delayed matching

- In (Cirstea et al., 2004), we studied a more permissive type system, still enforcing subject reduction, but allowing to typecheck some terms with infinite reductions. Therefore, this type system was not fit for using the $\rho$-calculus as a proof-term language.

- The type systems of (Cirstea et al., 2001; Barthe et al., 2003) were designed in order to provide a strongly normalizing calculus where there was no restriction on the type of the constants (apart those imposed by the type system). Until now, strong normalization was an open problem for these systems. Here, we show this property for a slight variation of (Barthe et al., 2003). We have introduced a signature $\boldsymbol{\Sigma}$ which prevents the type of a constant to depend on free variables.

In rules (MSORT) and (PROD), the first premise avoids a collapse of the $P^{2} T S$ cube. If we had just taken $\Psi_{0}: s_{1}$, with $\vdash_{\rho} f: \boldsymbol{\Pi}(\beta: *)$. $\beta$, the pattern $f \cdot \gamma$ would have sort $*$ but could be used to instantiate the type variable $\gamma$, enabling polymorphism in the simply-typed system.

In the rule (Var), we use $\Gamma_{\square} \triangleq\left\{X: \Phi \in \Gamma \mid \Sigma, \Gamma \vdash_{\rho} \Phi: \square\right\}$ to avoid free term variables occuring in the type of a variable. It is mainly because we want to keep the system "simply-typed", in the sense that matching constraints occurring in types do not yield types depending on terms. For the type systems allowing terms depending on types, this restriction will have to be relaxed.

Finally, the rule (STRUCT) can seem quite restrictive, since case-dependent expressions such as $\boldsymbol{\lambda}(0: n a t) .0 ; \boldsymbol{\lambda}\left(\boldsymbol{s}^{\bullet} \boldsymbol{X}: \mathbf{n a t}\right) . X$ are forbidden. However, it is non-trivial to weaken this rule. For example, if we had typed $\lambda(0$ : nat $) .0 ; \lambda(s \bullet X:$ nat $) . X$ with $\Pi(N:$ nat $) . n a t$, we could have built a typed term with infinite reductions as in (Cirstea et al., 2004).

The notion of arity we have assumed in the untyped encoding can be properly defined here using types: if $f_{i}$ has type $\Phi_{i}$, then $\alpha_{i}$ is defined as $\alpha\left(\Phi_{i}\right)$ :

$$
\begin{aligned}
\alpha(\Xi) & \triangleq \\
\alpha(\Pi P . \Psi) & \triangleq 1+\alpha(\Psi) \\
\alpha([P \ll B] \Psi) & \triangleq \alpha(\Psi)
\end{aligned}
$$




$$
\begin{aligned}
& \overline{\emptyset \vdash_{\rho} *: \square} \text { (Ахіом) } \frac{\Sigma, \Gamma \vdash_{\rho} A: \Phi \quad \Sigma, \Gamma \vdash_{\rho} B: \Phi}{\Sigma, \Gamma \vdash_{\rho} A ; B: \Phi} \text { (StruCT) } \\
& \frac{\Sigma, \Gamma_{\square} \vdash_{\rho} \Phi: s \quad X \notin \mathcal{D o m}(\Gamma)}{\Sigma, \Gamma, X: \Phi \vdash_{\rho} X: \Phi}(\mathrm{VAR}) \frac{\Sigma \vdash_{\rho} \Phi: s \quad f \notin \mathcal{D o m}(\Sigma)}{\Sigma, f: \Phi \vdash_{\rho} f: \Phi} \text { (CoNST) } \\
& \frac{\Sigma, \Gamma \vdash_{\rho} A: \Phi \quad \Sigma, \Gamma \vdash_{\rho} \Psi: s \quad X \notin \operatorname{Dom}(\Gamma)}{\Sigma, \Gamma, X: \Psi \vdash_{\rho} A: \Phi}(\text { WEAK } \Gamma) \\
& \frac{\Sigma \vdash_{\rho} A: \Phi \quad \Sigma \vdash_{\rho} \Psi: s \quad f \notin \operatorname{Dom}(\Sigma)}{\Sigma, f: \Psi \vdash_{\rho} A: \Phi}(\text { WEAK } \Sigma) \\
& \frac{\Sigma, \Gamma \vdash_{\rho} A: \Psi \quad \Sigma, \Gamma \vdash_{\rho} \Phi: s \quad \Phi==_{\rho \infty} \Psi}{\Sigma, \Gamma \vdash_{\rho} A: \Phi} \text { (Conv) } \\
& \frac{\Sigma, \Gamma, \Delta \vdash_{\rho} A: \Phi \quad \Sigma, \Gamma \vdash_{\rho} \Pi(P: \Delta) \cdot \Phi: s}{\Sigma, \Gamma \vdash_{\rho} \lambda(P: \Delta) \cdot A: \Pi(P: \Delta) \cdot \Phi}(\mathrm{ABS}) \\
& \frac{\Sigma, \Gamma \vdash_{\rho} A: \Pi(P: \Delta) . \Phi \quad \Sigma, \Gamma \vdash_{\rho}[P \ll \Delta B] \Phi: s}{\Sigma, \Gamma \vdash_{\rho} A \cdot B:[P \ll \Delta B] \Phi} \text { (APPL) } \\
& \frac{\Sigma, \Gamma, \Delta \vdash_{\rho} A: \Phi \quad \Sigma, \Gamma \vdash_{\rho}[P \ll \Delta B] \Phi: s}{\Sigma, \Gamma \vdash_{\rho}[P \ll \Delta B] A:[P \ll \Delta B] \Phi}(\mathrm{MATCH}) \\
& \frac{\forall(X: \Psi) \in \Delta, \Sigma, \Gamma, \Delta \vdash_{\rho} \Psi: s_{1} \quad \Sigma, \Gamma, \Delta \vdash_{\rho} P: \Psi_{0} \quad \Sigma, \Gamma, \Delta \vdash_{\rho} \Phi: s_{2}}{\Sigma, \Gamma \vdash_{\rho} \Pi(P: \Delta) \cdot \Phi: s_{2}} \text { (PROD) } \\
& \forall(X: \Psi) \in \Delta, \Sigma, \Gamma, \Delta \vdash_{\rho} \Psi: s_{1} \\
& \frac{\Sigma, \Gamma, \Delta \vdash_{\rho} P: \Psi_{0} \quad \Sigma, \Gamma \vdash_{\rho} B: \Psi_{0} \quad \Sigma, \Gamma, \Delta \vdash_{\rho} \Phi: s_{2}}{\Sigma, \Gamma \vdash_{\rho}[P \ll \Delta B] \Phi: s_{2}} \text { (MSORT) }
\end{aligned}
$$

In the simply-typed system, $\left(s_{1}, s_{2}\right)=(*, *)$.

\section{Figure 4. The typing rules of $P^{2} T S$}

One is easily convinced that a term $f_{\boldsymbol{i}} \cdot \vec{A}$ where $\vec{A}$ contains more than $\alpha_{\boldsymbol{i}}$ elements can not be correctly typed. Similarly, in a term $(A ; B) \cdot \vec{C}, A$ and $B$ have a common type $\boldsymbol{\Phi}$ so $\vec{C}$ can not contain more than $\alpha(\Phi)$ elements.

The second condition on arities is enforced too: in a given matching equation $f_{i} \cdot \vec{P}_{(1 . . p)} \ll f_{j} \cdot \vec{B}_{(1 . . q)}$, typing enforces that $f_{i} \cdot \vec{P}_{(1 . . p)}$ and $f_{j} \cdot \vec{B}_{(1 . . q)}$ have the same type, which immediately imposes $\alpha_{i}-p=\alpha_{j}-q$.

Some properties of these calculi, proved in (Barthe et al., 2003), are:

Lemma 2 (Substitution) If $\boldsymbol{\Gamma}, \boldsymbol{X}: \boldsymbol{\Phi}, \Delta \vdash_{\rho} \boldsymbol{A}: \boldsymbol{\Psi}$ and $\boldsymbol{\Gamma} \vdash_{\rho} \boldsymbol{B}: \boldsymbol{\Phi}$, then $\Gamma, \Delta[X:=B] \vdash_{\rho} A[X:=B]: \Psi[X:=B]$.

\section{THEOREM 2 (SUBJECT REDUCTION)}

$$
\text { If } \Gamma \vdash_{\rho} A: \Phi \text { and } A \mapsto A^{\prime} \text {, then } \Gamma \vdash_{\rho} A^{\prime}: \Phi \text {. }
$$

LEMMA 3 (UNIQUENESS OF TYPES UP TO SECOND ORDER) If $\left(s_{1}, s_{2}\right) \in\{(*, *),(\square, *)\}$, if $\Gamma \vdash_{\rho} A: \Phi_{1}$ and $\Gamma \vdash_{\rho} A: \Phi_{2}$, then $\Phi_{1} \bar{p}_{p \infty} \Phi_{2}$.

In this paper, we only treat the case of the simply typed calculus, corresponding to $\left(s_{1}, s_{2}\right)=\{(*, *)\}$. In particular, this implies uniqueness of types. 
As a conclusion to this section, let us briefly explain why usual reducibility techniques seem to fail for this typed calculus. Roughly speaking, the interpretation of a type $\boldsymbol{\Pi}(P: \Delta) . \Phi$ should be a function space whose domain is defined not only as the interpretation of the type of $P$ but also as terms matching with $P$ and whose suitable subterms belong to the interpretations of the types appearing in $\Delta$. Quickly, this imbrication of interpretations leads to circularities in the definition of interpretations. Thus, it seems really tricky to obtain a proper definition of the reducibility candidates.

\section{The System $F \boldsymbol{\omega}$}

In this section, we shortly recall the type system $F \omega$, first introduced and studied in (Girard, 1972). The formalism and its properties have been generalized to the Calculus of Constructions (Coquand and Huet, 1988), and later on to Pure Type Systems. Here, we follow the generic presentation of (Barendregt, 1992). The inference rules are given in Fig. 5. Here, the possible product rules are $\{(*, *),(\square, *),(\square, \square)\}$.

$$
\begin{aligned}
& \overline{\emptyset \vdash_{\mathrm{F} \omega} *: \square} \text { (AXIOM) } \frac{\Gamma, x: \sigma \vdash_{\mathrm{F}_{\omega}} t: \tau \quad \Gamma \vdash_{\mathrm{F} \omega} \Pi(x: \sigma) \cdot \tau: s}{\Gamma \vdash_{\mathrm{F} \omega} \lambda(x: \sigma) \cdot t: \Pi(x: \sigma) \cdot \tau} \text { (ABS) }
\end{aligned}
$$

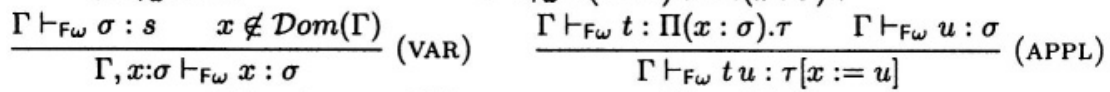

$$
\begin{aligned}
& \frac{\Gamma \vdash_{\mathrm{F}_{\omega}} t: \sigma \quad \Gamma \vdash_{\mathrm{F}_{\omega}} \tau: s \quad x \notin \operatorname{Dom}(\Gamma)}{\Gamma, x: \tau \vdash_{\mathrm{F}_{\omega}} t: \sigma} \text { (WEAK) } \\
& \frac{\Gamma \vdash_{\mathrm{F}_{\omega}} t: \tau \quad \Gamma \vdash_{\mathrm{F}_{\omega}} \sigma: s \quad \sigma={ }_{\beta} \tau}{\Gamma \vdash_{\mathrm{F}_{\omega}} t: \sigma}(\mathrm{CONV}) \\
& \frac{\Gamma \vdash_{\mathrm{F}_{\omega}} \sigma: s_{1} \quad \Gamma, x: \sigma \vdash_{\mathrm{F}_{\omega}} \tau: s_{2} \quad\left(s_{1}, s_{2}\right) \in\{(*, *),(\square, *),(\square, \square)\}}{\Gamma \vdash_{\mathrm{F}_{\omega}} \Pi(x: \sigma) \cdot \tau: s_{2}} \text { (PROD) }
\end{aligned}
$$

Figure 5. The typing rules of $F_{\omega}$

In all the remaining, for a type $\Pi(x: \sigma) . \tau$, we will use the usual type arrow abbreviation $\sigma \rightarrow \tau$ whenever $x \notin \mathcal{F} \mathcal{V}(\tau)$, i.e. for terms depending on terms (product rule $(*, *)$ ) and for types depending on types (product rule $(\square, \square)$ ).

Some well-known properties of this calculus are (Girard, 1972; Barendregt, 1992):

Lemma 4 (Substitution) If $\Gamma, x: \sigma, \Delta \vdash_{\mathrm{F}_{\omega}} t: \tau$ and $\Gamma \vdash_{\mathrm{F}_{\omega}} u: \sigma$, then $\Gamma, \Delta[x:=u] \vdash_{F w} t[x:=u]: \tau[x:=u]$.

THEOREM 3 (SUBJECT REDUCTION)

If $\Gamma \vdash_{F_{\omega}} t: \sigma$ and $t \mapsto_{\beta} t^{\prime}$, then $\Gamma \vdash_{\mathrm{F}_{\omega}} t^{\prime}: \sigma$.

LEMMA 5 (UNIQUENESS OF TYPES)

If $\Gamma \vdash_{\mathrm{F}_{\omega}} t: \sigma_{1}$ and $\Gamma \vdash_{\mathrm{F}_{\omega}} t: \sigma_{2}$, then $\sigma_{1}={ }_{\beta} \sigma_{2}$.

THEOREM 4 (STRONG NORMALIZATION)

If $\Gamma \vdash_{\mathbf{F} \omega} t: \sigma$, then $t$ is strongly normalizing.

\section{The typed translation algorithm}

Here, instead of translating a term to a term, we translate a typed term into a (typable) term. For simplicity of presentation, we still write $\llbracket A \rrbracket$ but, as one can see on 
Fig. 6, the translation of a term $A$ is generally based on the fact that $A$ is typable. Supposing we are given a type derivation for a judgment $\Sigma, \Gamma \vdash_{\rho} A: \Phi$, we recursively build a term $\llbracket A \rrbracket$ typable in $\llbracket \Gamma \rrbracket$. There is no translation for $\boldsymbol{\Sigma}$ since, as we will see in Section 7 , the context $x_{\perp}: \perp$. is sufficient to type $\|f\|$ for any constant $f \in \Sigma$.

For the rest of the paper, we adopt the following abbreviations, for any types $\sigma, \sigma_{1}$, $\ldots, \sigma_{n}, \tau$ in $\mathbf{F} \omega$. The third definition is a special case of the second one with $\alpha=0$ :

$$
\begin{array}{ll}
{[\sigma]^{S} \rightarrow \tau} & \triangleq \underbrace{\sigma \rightarrow \ldots \rightarrow \sigma}_{S} \rightarrow \tau \\
\left\{\sigma_{1}, \ldots, \sigma_{\alpha}\right\} & \triangleq \Pi(\beta: *) \cdot\left(\left[\sigma_{1} \rightarrow \ldots \sigma_{\alpha} \rightarrow \beta\right]^{S} \rightarrow \beta\right) \\
\{\emptyset\} & \triangleq \Pi(\beta: *) \cdot\left([\beta]^{S} \rightarrow \beta\right)
\end{array}
$$

For each variable $X$ appearing in a $P^{2} T S$ term, we add in the corresponding $\lambda$-term a type variable $\beta_{\boldsymbol{X}}$ which appears in the type of $\llbracket X \rrbracket$. This variable $\beta_{\boldsymbol{X}}$ is common to every occurrence of $X$ in the term, and if $X$ is bound, we bind $\beta_{\boldsymbol{X}}$ at the same point as $X$ in the translation. If $X$ is free, then in the translation of the context, the type variable $\boldsymbol{\beta}_{\boldsymbol{X}}$ appears just before $X$. The need for $\boldsymbol{\beta}_{\boldsymbol{X}}$ is explained in Section 7.

First we define the translation of types (i.e. terms such that $\Gamma r_{\rho} \Phi: *$ ) by four mutually dependent definitions:

$\llbracket \Phi \rrbracket \frac{X}{\gamma}$ translates the type $\Phi$, supposing it is the type of the variable $X$ depending on the list of type variables $\vec{\gamma}$. The free type variable $\beta_{\boldsymbol{X}}$ (univocally corresponding to $X$ ) appears in this translation.

$$
\begin{aligned}
& \llbracket \Xi \rrbracket_{\vec{\gamma}}^{X} \triangleq \beta_{X} \vec{\gamma} \quad \text { (where } \Xi \text { is atomic) } \\
& \llbracket \Pi(P: \Delta) \cdot \Psi \rrbracket \frac{X}{\vec{\gamma}} \triangleq \prod_{\left(Y: \Phi_{Y}\right) \in \Delta} \overrightarrow{\beta_{Y}: \mathbb{K}\left(\Phi_{Y}\right)_{\mathbb{\sigma}}} \cdot\left(\left[P P_{\lrcorner \Delta} \rightarrow \llbracket \Psi \rrbracket_{\vec{\gamma} \cup \overrightarrow{\beta_{Y}}}^{X}\right)\right. \\
& \llbracket\left[P \ll \Delta B \mid \Psi \rrbracket \frac{X}{\gamma} \triangleq \prod_{\left(Y: \Phi_{Y}\right) \in \Delta} \overrightarrow{\beta_{Y}^{\prime}: \mathbb{K}\left(\Phi_{Y}\right)_{\theta}} \cdot\left(\left(\sigma \rightarrow[P\lrcorner_{\Delta \Delta}\right) \rightarrow \llbracket \Psi \rrbracket_{\vec{\gamma} \cup \overrightarrow{\beta_{Y}}}^{X}\right)\right. \\
& \text { where } \llbracket \Gamma \rrbracket \vdash_{F \omega} \llbracket B \rrbracket: \sigma
\end{aligned}
$$

$\llbracket \Phi \rrbracket \frac{f}{\vec{\tau}}$ translates the type $\Phi$, supposing it is the type of the constant $f$ depending on the list of types $\vec{\tau}$.

$$
\begin{aligned}
& {[\Xi]_{\vec{\tau}}^{f} \triangleq\{\vec{\tau}\} \quad \text { (where } \Xi \text { is atomic) }}
\end{aligned}
$$

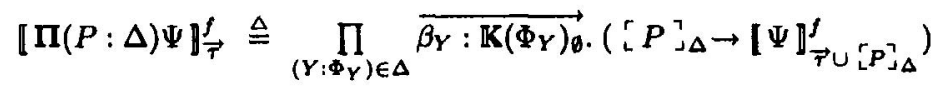

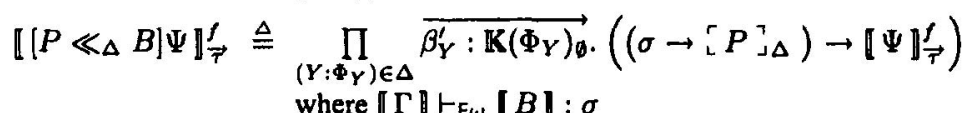

The only free variable appearing in $\llbracket \Phi \rrbracket_{\emptyset}^{X}$ is $\beta_{X}$, and the arguments $\vec{\gamma}$ of $\beta_{\boldsymbol{X}}$ are the bound variables whose scope extends to this subterm of the type. Similarly, in $\llbracket \Phi \rrbracket_{\emptyset}^{f}$, no variable is free, and all the bound variables whose scope extends to the subterm $\{\vec{\tau}\}$ are represented in this subterm. 
$\left[{ }_{[}\right\urcorner_{{ }_{\Delta}}$ flattens a pattern $P$ with $\mathcal{F V}(P) \subseteq \mathcal{D}$ om $(\Delta)$. Since patterns appear in the $P^{2} T S$ types, the translation at the type level must be accurate.

$$
\begin{aligned}
& {\left[f_{i} \cdot \vec{P}\right]_{\Delta \Delta} \triangleq \llbracket \Pi\left(P_{p+1}^{\prime}: \Delta_{p+1}\right) \ldots \Pi\left(P_{\alpha_{i}}^{\prime}: \Delta_{\alpha_{i}}\right) \cdot \Xi \rrbracket \frac{f}{\left[P_{\Delta \Delta}^{\urcorner}\right.}} \\
& \text {where } \Sigma \vdash_{\rho} f_{i}: \Pi\left(P_{1}^{\prime}: \Delta_{1}\right) \ldots \Pi\left(P_{\alpha_{i}}^{\prime}: \Delta_{\alpha_{i}}\right) . \Xi \\
& \left.[X]_{\lrcorner \Delta} \triangleq \llbracket \Phi\right]_{\theta}^{X} \quad \text { if } X: \Phi \in \Delta
\end{aligned}
$$

$\mathbb{K}(\Phi)_{\vec{k}}$ computes the kind of $\beta_{X}$ if $X$ has type $\Phi$.

$$
\begin{aligned}
\mathbb{K}(\Xi)_{\vec{k}} & \triangleq \vec{k} \rightarrow * \\
\mathbb{K}(\Pi(P: \Delta) \cdot \Psi)_{\vec{k}} & \triangleq \mathbb{K}(\Psi)_{\vec{k} \cup} \underset{\left(Y: \Phi_{Y}\right) \in \Delta}{\cup} \mathbf{K}\left(\Phi_{Y}\right) \\
\mathbb{K}([P \ll \Delta B] \Psi)_{\vec{k}} & \triangleq \mathbb{K}(\Psi)_{\vec{k} \cup} \underset{\left(Y: \Phi_{Y}\right) \in \Delta}{\cup} \mathbf{K}\left(\Phi_{Y}\right)
\end{aligned}
$$

We can extend this translation to contexts, the base case being given by $x_{\perp}$ :

$$
\begin{aligned}
\llbracket \emptyset \rrbracket & \triangleq x_{\perp}: \perp \\
\llbracket \Gamma, X: \Phi \rrbracket & \left.\triangleq \llbracket \Gamma \rrbracket, \beta_{X}: \mathbb{K}(\Phi)_{\emptyset}, X: \llbracket \Phi \rrbracket_{\emptyset}^{X} \quad \text { (if } \Gamma \vdash_{\rho} \Phi: *\right) \\
\llbracket \Gamma, X: * \rrbracket & \triangleq \llbracket \Gamma \rrbracket, X: *
\end{aligned}
$$

Finally, we can translate typed terms. The translation is given in two distinct parts: in Fig. 6, we give all the cases that are simply adapted from the untyped case. In Fig. 7, we deal with the trickiest situations: matching constraints and conversion in the types. These last cases are further explained in Section 7.

\section{Rationale of the typed translation}

In this section we treat three key constructs of the typed translation:

1 the type of a translated constant (accounting for the use of System F);

2 the type of a variable (requiring types depending on types);

3 the translation of matching constraints appearing in the $P^{2} T S$ types.

\section{Typing the translation of a constant}

First, let us study how constants and their translation affect typing. In order to get a typed translation, in the previous section, we have added to the untyped term $\llbracket f_{i} \rrbracket$ some type abstractions. The type abstractions $\lambda\left(\beta_{Y}: \mathbb{K}\left(\Phi_{Y}\right)_{\emptyset}\right)$ are needed for correctly typing the variables, as we will see in the next subsection. Here, we are interested in the type abstraction $\lambda(\beta: *)$ appearing in $\llbracket f_{i} \rrbracket$.

To explain the modifications we made, let us start from the untyped translation. We supposet $f_{i}: \boldsymbol{\Pi} P_{1} \ldots \boldsymbol{\Pi} P_{\alpha_{i}}, \Xi$ where $\boldsymbol{\Xi}$ is an atomic type, and we assume that each $P_{n}$ is translated to a certain type $\sigma_{n}$. Then we have:

$$
x_{\perp}: \beta \vdash \llbracket f_{i} \rrbracket: \sigma_{1} \rightarrow \ldots \rightarrow \sigma_{\alpha} \rightarrow\left[\sigma_{1} \rightarrow \ldots \rightarrow \sigma_{\alpha} \rightarrow \beta\right]^{S} \rightarrow \beta
$$

What remains unclear is the meaning of $\beta$. The type of a translated abstraction is:

$$
\vdash \llbracket \lambda\left(f_{i} \cdot \vec{X}_{(1 . p)}\right) . A \rrbracket:\left(\sigma_{p+1} \rightarrow \ldots \rightarrow \sigma_{\alpha} \rightarrow\left[\sigma_{1} \rightarrow \ldots \rightarrow \sigma_{\alpha} \rightarrow \tau\right]^{S} \rightarrow \gamma\right) \rightarrow \gamma
$$




$$
\begin{aligned}
& \llbracket X \rrbracket \triangleq X \\
& \llbracket f_{i} \rrbracket \triangleq \lambda \overrightarrow{\beta_{Y}}, \lambda x_{1} \ldots \lambda \overrightarrow{\beta_{Y}} \alpha_{i} \cdot \lambda x_{\alpha_{i}} \cdot \lambda(\beta: *)\left(\lambda \vec{z}_{(1 . . S)} .\left(z_{i} \vec{x}_{\left(1 \ldots \alpha_{i}\right)}\right)\right) \\
& \text { where } \Sigma \vdash_{\rho} f_{i}: \Pi\left(P_{1}: \Delta_{1}\right) \ldots \Pi\left(P_{\alpha_{i}}: \Delta_{\alpha_{i}}\right) . \Xi \\
& \text { and } \vec{\beta}_{n} \text { correspond to } \overrightarrow{\left(Y: \Phi_{Y}\right)} \in \Delta_{n} \text {, with } \overrightarrow{\beta_{Y}: \mathbb{K}\left(\Phi_{Y}\right)_{\emptyset}} \text {. }
\end{aligned}
$$

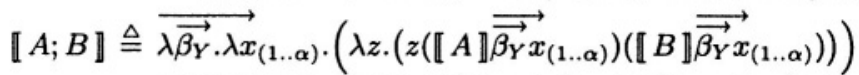

$$
\begin{aligned}
& \text { where } \Sigma, \Gamma \vdash_{\rho} A ; B: \Pi\left(P_{1}: \Delta_{1}\right) \ldots \Pi\left(P_{\alpha_{i}}: \Delta_{\alpha_{i}}\right) . \Xi \\
& \text { and } \overrightarrow{\beta_{Y}} \text { are the type variables corresponding to } \mathcal{F V}\left(P_{n}\right) \text {. } \\
& \llbracket \lambda(X: \Phi) \cdot A \rrbracket \triangleq \lambda\left(\beta_{X}: \mathbb{K}(\Phi)_{\emptyset}\right) \cdot \lambda\left(X: \llbracket \Phi \rrbracket_{\emptyset}^{X}\right) \cdot \llbracket A \rrbracket \\
& \text { where } \Sigma, \Gamma \vdash_{\rho} \lambda(X: \Phi) \cdot A: \Pi(X: \Phi) \cdot \Psi \\
& \llbracket \lambda\left(f_{i} \cdot \vec{P}_{(1 . . p)}: \Delta\right) \cdot A \rrbracket \triangleq \lambda_{X \in \Delta}\left(\overrightarrow{\beta_{X}: \mathbb{K}\left(\Phi_{X}\right)}\right) \cdot \lambda y \cdot\left(y \vec{\perp}{\left.\overrightarrow{\left(x _ { \perp } \left\lceilP^{\prime}{ }^{\jmath} \Delta\right.\right.}\left[\beta_{Y}:=\perp\right]\right)}_{\left(p+1 \ldots \alpha_{i}\right)}\right. \\
& \left.\tau \overrightarrow{\left(x_{\perp} \tau_{0}\right)} \llbracket \lambda \overrightarrow{P: \Delta}(1 . . p) \cdot \lambda \overrightarrow{x^{\prime}:\left[P^{\prime}\right]_{\lrcorner \Delta}\left(p+1 \ldots \alpha_{i}\right)} \cdot A \rrbracket \overrightarrow{\left(x_{\perp} \tau_{0}\right)}\right) \\
& \text { where } \Sigma, \Gamma \vdash_{\rho} \lambda\left(f_{i} \cdot \vec{P}_{(1 . . p)}: \Delta\right) \cdot A: \Pi\left(f_{i} \cdot \vec{P}_{(1 . . p)}: \Delta\right) . \Psi
\end{aligned}
$$

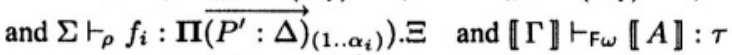

$$
\begin{aligned}
& \text { and } \llbracket \Gamma \rrbracket \vdash_{F} \llbracket \lambda \vec{P}_{(1 \ldots p)} \cdot \lambda \vec{x}_{\left(p+1 \ldots \alpha_{i}\right)} \cdot A \rrbracket: \tau_{0}
\end{aligned}
$$

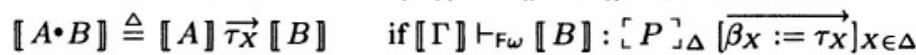

$$
\begin{aligned}
& \text { where } \Sigma, \Gamma \vdash_{\rho} A \cdot B:[P \ll \Delta B] \Psi \\
& \llbracket[P \ll \Delta B] A \rrbracket \triangleq \text { the term obtained by head- } \beta \text {-reducing } \llbracket(\lambda(P: \Delta) \cdot A) \cdot B \rrbracket
\end{aligned}
$$

Figure 6. Typed term translation without matching constraints

Therefore, the $\lambda$-term $\llbracket \lambda\left(f_{i} \cdot \vec{X}_{(1 . . p)}\right) \cdot A \rrbracket\left(\llbracket f_{i} \rrbracket \llbracket B_{1} \rrbracket \ldots \llbracket B_{p} \rrbracket\right)$ has a valid type only if $\left[\sigma_{1} \rightarrow \ldots \rightarrow \sigma_{\alpha} \rightarrow \tau\right]^{S} \rightarrow \gamma=\left[\sigma_{1} \rightarrow \ldots \rightarrow \sigma_{\alpha} \rightarrow \beta\right]^{S} \rightarrow \beta$, i.e. $\tau=\beta=\gamma$ and $\vdash \llbracket B_{1} \rrbracket: \sigma_{1} \ldots \vdash \llbracket B_{p} \rrbracket: \sigma_{p}$. The types $\beta$ and $\gamma$ should be replaced by the return type $\tau$ of the function which is applied to $\llbracket f_{i} \cdot \vec{B} \rrbracket$. Since one can not guess what function will be applied to a given term, we introduce the polymorphism of Girard's System $F$ in the target language. The resulting modification can be seen on Fig. 6: in $\llbracket f_{i} \rrbracket$, we abstract over the type variable $\beta$, which is instantiated with $r$ by $\llbracket \lambda\left(f_{i} \cdot \vec{P}\right) . A \rrbracket$.

Thanks to polymorphism, the variable $\boldsymbol{x}_{\perp}$ can get type $\Pi(\iota: *) . \iota$, which is usually noted $\perp$. Then, if we need an arbitrary term with type $\sigma$, we use $\boldsymbol{x}_{\perp} \sigma$. This means that all the $\lambda$-terms we build are typable in a context containing $x_{\perp}: \perp$; again, we can add an abstraction " $\lambda\left(\boldsymbol{x}_{\perp}: \perp\right)$ " to get a closed term.

The types $\llbracket \Phi \rrbracket_{\emptyset}^{f}$ have been built to fit with the new translation of constants: a translated constant $\llbracket f_{i} \rrbracket$ with arity $\alpha_{i}$ takes $\alpha_{i}$ arguments with types $\sigma_{1} \ldots \sigma_{\alpha_{i}}$ and returns a term with type $\left\{\sigma_{1}, \ldots \sigma_{\alpha_{i}}\right\}$. The types $\left[P_{\lrcorner \Delta}\right.$ extend this notion to nested patterns: for instance $\llbracket f \cdot\left(g \cdot x_{1}\right) \cdot x_{2} \rrbracket$ will have type $\left\{\left\{\sigma_{1}\right\}, \sigma_{2}\right\}$. This flattening process keeps the shape of the pattern but forgets the constants used.

\section{Typing a variable}

In this subsection, we explain why we need a new type variable $\boldsymbol{\beta}_{\boldsymbol{X}}$ for each variable $X$ appearing in a $P^{2} T S$ term (including bound variables appearing in a type). 
- (Constraint postponement): if $\nexists \overrightarrow{\tau_{X}}, \sigma={ }_{\beta}\left[P_{\nu_{\Delta}}^{\urcorner}\left[\overrightarrow{\beta_{X}:=\tau_{X}}\right]_{X \in \operatorname{Dom}(\Delta)}\right.$

$$
\begin{aligned}
\llbracket A \cdot B \rrbracket \triangleq & \left.\lambda_{(Y \in \Delta)} \overrightarrow{\beta_{Y}^{\prime}: \mathbb{K}\left(\Phi_{Y}\right)} \cdot \lambda\left(w: \sigma \rightarrow{ }_{L} P_{\left\lfloor Y:=Y^{\prime} \mid\right.}\right\lrcorner_{\Delta}\right) \cdot\left(\llbracket A \rrbracket \overrightarrow{\beta_{Y}^{\prime}}(w \llbracket B \rrbracket)\right) \\
& \text { where } \Sigma, \Gamma \vdash_{\rho} A \cdot B:[P \ll \Delta B] \Psi \\
& \text { and } \llbracket \Gamma \rrbracket \vdash_{F_{\omega}} \llbracket B \rrbracket: \sigma
\end{aligned}
$$

- (Constraint resolution) : For a postponement variable $w: \sigma \rightarrow{ }_{\mathrm{L}}^{r} P{ }^{\urcorner} \Delta$ appearing in a term $\llbracket A \rrbracket$, whenever a subsequent instantiation $\theta$ of some free type variables (in $\sigma$ ) enforces:

$$
\exists \overrightarrow{\tau_{X}}, \sigma \theta=\beta \quad\left\ulcorner P{ }_{\lrcorner \Delta}^{\urcorner}\left[\overrightarrow{\beta_{X}:=\tau_{X}}\right]_{X \in \mathcal{D} o m(\Delta)}\right.
$$

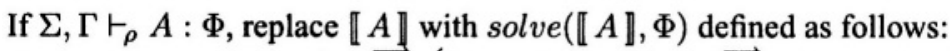

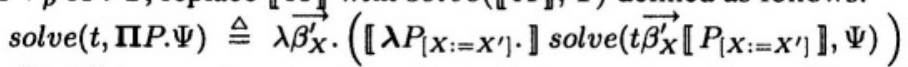

(where $\llbracket \lambda P . \rrbracket$ denotes the same abstractions as in Fig. 6 when encoding $\llbracket \lambda P . A \rrbracket$ )

$$
\begin{aligned}
& \text { solve }(t,[P \ll \Delta B] \Psi) \triangleq \lambda_{Y \in \mathcal{D o m}(\Delta)} \overrightarrow{\beta_{Y}^{\prime \prime}} \cdot \lambda w^{\prime} \text {. solve }\left(t \overrightarrow{\beta_{Y}^{\prime \prime}} w^{\prime}, \Psi\right) \\
& \text { if } P \ll B \text { is not the matching constraint associated to } w \text {. } \\
& \text { solve }(t,[P \ll \Delta B] \Psi) \triangleq t \overrightarrow{\tau_{X}}(\lambda(x: \sigma \theta) . x) \\
& \text { if } P \ll B \text { is the matching constraint associated to } w \text {. }
\end{aligned}
$$

Figure 7. Typed term translation for delayed matching constraints

Consider the following examples:

$$
\begin{array}{rr}
(\Xi: *),(X: \Pi(Y: \Xi) . \Xi) \vdash_{\rho} X & : \Pi(Y: \Xi) . \Xi \\
(\Xi: *) \vdash_{\rho} \lambda(Y: \Xi) . Y: \Pi(Y: \Xi) . \Xi \\
(\Xi: *),(f: \Pi(Y: \Xi) . \Xi) \vdash_{\rho} f & : \Pi(Y: \Xi) . \Xi
\end{array}
$$

Both terms $\boldsymbol{\lambda} \boldsymbol{Y} . \boldsymbol{Y}$ and $\boldsymbol{f}$ can instantiate $X$ since they have the same type. However, the typed translation gives (in the context $\Gamma=\left(x_{\perp}: \perp\right)$ ):

$$
\begin{array}{ll}
\Gamma \vdash_{F \omega} \lambda\left(\beta_{Y}: *\right) . \lambda\left(Y: \beta_{Y}\right) . Y: \Pi\left(\beta_{Y}: *\right) \cdot \beta_{Y} \rightarrow \beta_{Y} \\
\Gamma \vdash_{F \omega} \llbracket f \rrbracket & : \Pi\left(\beta_{Y}: *\right) . \beta_{Y} \rightarrow\left\{\beta_{Y}\right\}
\end{array}
$$

The type variable $\beta_{X}$ which appears in $\vdash_{\mathrm{F} \omega} X: \Pi\left(\beta_{Y}: *\right) . \beta_{Y} \rightarrow \beta_{X} \beta_{Y}$ allows us to treat both cases: an abstraction $\lambda X$. $A$ is translated into $\lambda \beta_{X} \cdot \lambda X . \llbracket A \rrbracket$, so we can give the expected type to $X$ if we instantiate $\beta_{X}$ with the correct term:

$$
\begin{array}{l|l|l}
\lambda Y . Y & \beta_{X}:=\lambda(\beta: *) . \beta & \beta_{X} \beta_{Y} \mapsto_{\beta} \beta_{Y} \\
\hline f & \beta_{X}:=\lambda(\beta: *) .\{\beta\} & \beta_{X} \beta_{Y} \mapsto \beta\left\{\beta_{Y}\right\}
\end{array}
$$

The need for types depending on types appears here: $\beta_{X}$ must be able to build a new type where some type variables, like $\beta_{Y}$, may appear whereas they are bound in the type of $X$. The function $\mathbb{K}(\cdot)$ computes a suitable kind for $\beta_{X}$ according to the kinds of the arguments $\beta_{Y}$ of $\beta_{X}$. Here, we have $\vdash_{F_{\omega}} \beta_{X}: * \rightarrow *$.

\section{Translating matching constraints appearing in $P^{2} T S$ types}

The part of the typed translation shown in Fig. 6 mainly consists in correctly combining information obtained by the translation of smaller terms. However, for application (and matching constraints), the argument of a function must transmit it some type 
information. In $\boldsymbol{P}^{\mathbf{2}} \boldsymbol{T S}$, this process is initiated by the matching constraints appearing in types, and carried on by the conversion rule.

In System $\mathbf{F} \boldsymbol{\omega}$, we can not encode pattern matching in the types, so matching constraints must be treated at the meta-level, i.e. during the translation. Let us study the two kinds of matching constraints appearing in the types:

$\left[P \ll_{\Delta} B\right] \Psi$ with $\exists \boldsymbol{\theta}, \boldsymbol{B} \overline{7}_{\boldsymbol{\alpha} \phi} \boldsymbol{P \theta}:$ By successive application of Lemmas 2, 1 and 4 , we can prove that the same equality holds for the types in System $\mathbf{F} \boldsymbol{\omega}$ : if $₫ B \rrbracket$ has type $\sigma$, then $\exists \overrightarrow{\tau_{X}}, \sigma=\beta\left[{ }_{L} P{ }_{\lrcorner \Delta}\left[\overrightarrow{\beta_{X}:=\tau_{X}}\right]\right.$ where $\vec{X}=\mathcal{F V}(P)$. The proof of Theorem 5 is constructive: it gives an algorithm for computing the $\overrightarrow{\tau_{X}}$.

$\left[P \ll_{\Delta} B\right] \Psi$ with $\forall \theta, B \neq_{\infty \phi} P \theta:$ In this case, a new postponement variable $\boldsymbol{w}$ is created with type $\sigma \rightarrow\left[P{ }^{\prime}{ }_{\Delta}\right.$, where $\sigma$ is the type of $\llbracket B \rrbracket$. The type of $w$ appears in $\llbracket[P \ll B] \Psi \rrbracket_{\varnothing}^{X}$, accounting for the delayed matching constraint in the type. The term $w \llbracket B \rrbracket$ is used so that the $\lambda$-term is well-typed, since $\llbracket \lambda(P: \Delta) \cdot A \rrbracket$ expects a term of type $[\boldsymbol{P}]_{\Delta \Delta}$. Suppose some subsequent applications instantiate some free variables in $B$ (replacing it with a term $\boldsymbol{B} \theta_{0}$ ) such that $\exists \theta, B \theta_{0}=\varnothing \theta$. Then, we should instantiate the free type variables $\overrightarrow{\beta_{\boldsymbol{X}}}$ of ${ }_{L} P_{\boldsymbol{J}_{\Delta}}^{\urcorner}$with suitable types $\overrightarrow{\boldsymbol{\tau}_{\boldsymbol{X}}}$ and instantiate $\boldsymbol{w}$ with the identity since we had translated $B$ into $w \llbracket B \rrbracket$.

From a typing point of view, it is sound: because of the substitutions $\theta_{0}$ and $\theta$, the type of $w$ is now $\sigma \llbracket \theta_{0} \rrbracket \rightarrow[P]_{\Delta}\left[\overrightarrow{\beta_{X}:=\tau_{X}}\right]$ and the equality $B \theta_{0}=\overline{p o s} P \theta$ ensures that $\sigma\left[\theta_{0} \rrbracket=\beta\left[P{ }_{\lrcorner \Delta}\left[\overrightarrow{\beta_{X}:=\tau_{X}}\right]\right.\right.$, which means $\boldsymbol{w}$ has a suitable type for identity. The subtle point is that $\boldsymbol{w}$ can be located quite deep in the term we are considering: this is why we use the function solve $(\cdot, \cdot)$ given in Fig. 7 , which performs a kind of $\eta$-expansion to instantiate $\boldsymbol{w}$.

\section{Strong normalization}

In this section, we give the properties of our typed encoding.

Proposition 1 (FAITHFul REDUCTIONS) Lemma 1 and Theorem 1 are still valid with the typed translation: each $\rho \sigma \delta$-reduction can be mimicked by at least one $\boldsymbol{\beta}$-reduction (and the postponement variables $\boldsymbol{w}$ only prevent unsuccessful matchings).

LEMMA 6 (WELL-KINDEDNESS)

$\forall \Sigma, \forall \Gamma, \forall \Phi, \quad \Sigma, \Gamma \vdash_{\rho} \Phi: * \Rightarrow \llbracket \Gamma \rrbracket, \beta_{X}: \mathbb{K}(\Phi)_{\emptyset} \vdash_{F_{\omega}} \llbracket \Phi \rrbracket_{\emptyset}^{X}: *$

TheOREM 5 (WeLl-TYPed TRANSLATION) $\forall \boldsymbol{\Sigma}, \Gamma, A, \boldsymbol{\Phi}$, if $\boldsymbol{\Sigma}, \Gamma \vdash_{\rho} A: \Phi$ then, for afresh variable $Z, \quad \exists \tau_{A}, \llbracket \Gamma \rrbracket \vdash_{\mathrm{F}_{\omega}} \llbracket A \rrbracket: \llbracket \Phi \rrbracket_{\emptyset}^{Z}\left[\beta_{\mathbf{Z}}:=\tau_{A} \rrbracket\right.$

THEOREM 6 (STRONG NORMALIZATION OF TYPABLE $P^{2} T S$ TERMS) $\forall \boldsymbol{\Sigma}, \Gamma, A, \boldsymbol{\Phi}$, if $\boldsymbol{\Sigma}, \Gamma \vdash_{\rho} A: \Phi$ then $A$ is strongly normalizing.

Proof: A $P^{2} T S$-typable term $A$ is translated into an $\mathbf{F} \boldsymbol{\omega}$-typable term which has no infinite reduction, so by Proposition 1, $A$ is strongly normalizing.

\section{Conclusion and perspectives}

We have proved strong normalization of the simply-typed $\boldsymbol{P}^{2} \boldsymbol{T S}$ by translating it into System $\mathbf{F} \boldsymbol{\omega}$. First, we have shown how to encode untyped syntactic pattern 
matching in the $\lambda$-calculus. Introducing types in the translation then proved an interesting challenge. One difficulty comes from the pattern matching occuring in the $P^{2} T S$ types, which calls for accurate adjustments in the translation. Another remarkable point is that the typing mechanisms of $P^{2} T S$ can be expressed only with the expressive power of System $F \omega$, which is rather surprising since we only deal with the simply-typed $P^{2} T S$. This fact leads us to think that, with the same product rules, the expressive power of $P^{2} T S$ is greater than the one of the $\lambda$-calculus.

An interesting development of this work would be to adapt the proof for the other type systems of $P^{2} T S$. In the long term, we expect to use $P^{2} T S$ as the base language for a powerful proof assistant combining the logical soundness of the $\lambda$-calculus and the computational power of the rewriting. This proof of strong normalization is a main stepstone for this research direction, since logical soundness is deeply related to strong normalization.

Acknowledgements Thanks to H. Cirstea, C. Kirchner and L. Liquori for the constant support and interest they put in this work; P. Blackburn for some useful insights about the typed $\lambda$-calculus; S. Salvati for many fruitful informal discussions about System F; F. Blanqui, G. Dowek and anonymous referees for their valuable comments.

Long version A detailed version of this article containing proofs and type derivations can be found at http://www. loria.fr/ wack/papers/rhoSN.ps.gz .

\section{References}

Barendregt, H. P. (1992). Lambda calculi with types. In Abramsky, S., Gabbay, D., and Maibaum, T., editors, Handbook of Logic in Computer Science. Clarendon Press.

Barthe, G., Cirstea, H., Kirchner, C., and Liquori, L. (2003). Pure Patterns Type Systems. In POPL 2003, New Orleans, USA. ACM.

Blanqui, F. (2001). Definitions by rewriting in the calculus of constructions. In LICS, pages 9-18.

Cirstea, H. and Kirchner, C. (2000). The typed rewriting calculus. In Third International Workshop on Rewriting Logic and Application, Kanazawa (Japan).

Cirstea, H., Kirchner, C., and Liquori, L. (2001). The Rho Cube. In Honsell, F., editor, FOSSACS, volume 2030 of $L N C S$, pages 166-180, Genova, Italy.

Cirstea, H., Liquori, L., and Wack, B. (2004). Rewriting calculus with fixpoints: Untyped and first-order systems. In TYPES'03, LNCS, Torino. To be published.

Coquand, T. (1992). Pattern matching with dependent types. In Informalproceedings workshop on types for proofs and programs, pages 71-84. Båstad, Suède.

Coquand, T. and Huet, G. (1988). The calculus of constructions. Information and Computation, 76:95-120.

Dowek, G., Hardin, T., and Kirchner, C. (2003). Theorem proving modulo, revised version. Rapport de Recherche 4861, INRIA.

Girard, J.-Y. (1972). Interprétation fonctionnelle et élimination des coupures de l'arithmetique d'ordre supérieur. $\mathrm{PhD}$ thesis, Université Paris VII.

Kesner, D., Puel, L., and Tannen, V. (1996). A typed pattern calculus. Information and Computation, 124(1):32-61.

Klop, J., van Oostrom, V., and van Raamsdonk, F. (1993). Combinatory reduction systems: introduction and survey.TCS, 121:279-308.

Werner, B. (1994). Une Théorie des Constructions Inductives. PhD thesis, Université Paris VII. 